\title{
FAST EDGE DETECTION APPROACH BASED ON GLOBAL OPTIMIZATION CONVEX MODEL AND SPLIT BREGMAN ALGORITHM
}

\author{
Yu JING ${ }^{1 *}$, Jianxin LIU ${ }^{1}$, Zhaoxia LIU ${ }^{1}$, Hongju CAO ${ }^{2,1}$ \\ ${ }^{1}$ School of Software, Dalian University of Foreign Languages, Dalian, Liaoning, China \\ ${ }^{2}$ Information Science and Technology College, Dalian Maritime University, Dalian, Liaoning, China \\ yj_yi1982@sohu.com \\ Abstract \\ Active contour model is a typical and effective closed edge detection algorithm, which has been widely \\ applied in remote sensing image processing. Since the variety of the image data source, the complexity of the \\ application background and the limitations of edge detection, the robustness and universality of active \\ contour model are greatly reduced in the practical application of edge extraction. This study presented a fast \\ edge detection approach based on global optimization convex model and Split Bregman algorithm. Firstly, \\ the proposed approach defined a generalized convex function variational model which incorporated the RSF \\ model's principle and Chan's global optimization idea and could get the global optimal solution. Secondly, a \\ fast numerical minimization scheme based on split Bregman iterative algorithm is employed for overcoming \\ drawbacks of noise and others. Finally, the curve evolves to the target boundaries quickly and accurately. The \\ approach was applied in real special sea ice SAR images and synthetic images with noise, fuzzy boundaries \\ and intensity inhomogeneity, and the experiment results showed that the proposed approach had a better \\ performance than the edge detection methods based on the GMAC model and RSF model. The validity and \\ robustness of the proposed approach were also verified.
}

Keywords: edge detection; active contour; global optimization; numerical minimization; split Bregman algorithm

\section{INTRODUCTION}

In recent years, marine disasters occurred frequently, such as oil spill, sea ice and red tide. All of these current problems have shown a severe harm to the ecological security in coastal waters, the sea's use for the public and the marine environment. The frequent occurrence of the serous environmental disasters has become the focus of public attention. In the case of global warming, sea ice disaster is one of the most serious marine disasters all over the word. The early detection and monitoring of sea ice are critical to the environmental protection $[4,11]$. Recently, remote sensing image processing technology has developed rapidly and has been widely used, thus it is possible to monitor and identify sea ice by means of remote sensing device.

The edge of the image contains the boundary characteristic information of the interested target, which widely exists between target and background; therefore edge detection is a significant basic work for image processing, such as image registration, image segmentation and pattern recognition. At present, researchers have done a lot about edge detection, but because of the diversity and complexity of the images acquired by remote sensing device, it is still hard to find a universal edge detection method so far. Therefore, edge detection is considered a classical problem and bottleneck in the field of image processing and pattern recognition [15].

The edge detection approaches can be generally classified as two kinds including non-closed methods and closed methods. The non-closed edge detection methods include classical differential operator method, the optimal operator method, fitting method, multi-scale edge detection method and the edge detection method based on fuzzy theory. As for the closed edge detection methods, one of the most popular and widely used models is active contour models (ACMs) [18]. ACMs methods have more obvious advantages than other edge detection methods [9, 10]: Firstly, the edge detection results based on ACMs can reach subpixel accuracy. Secondly, ACMs can be easily formulated by introducing a variety of prior knowledge into the minimization energy function. Thirdly, ACMs is very robust for edge break and image noise. Finally, ACMs can provide closed and smooth contours, thus avoiding the post-processing in the non-closed edge detection methods. Cremers et al. [5] and $\mathrm{He}$ et al. [8] reviewed the major ACMs methods. Active Contour without Edges [2] is a simplified Mumford-Shah (MS) model [13], and it is considered as one of the most popular ACMs. An efficient region scalable fitting (RSF) energy model was proposed by Li et al. RSF model can deal with the problems of noise and intensity inhomogeneity [12]. Wang et al. proposed a novel active contour model using the superiority of RSF 
model and CV model for image segmentation [16]. Nevertheless, all of these ACMs have common problems, i.e., local minima and time consuming.

In this study, a general and fast edge detection approach based on global optimization convex model and Split Bregman algorithm was proposed. Firstly, the proposed approach defined a generalized convex function variational model which incorporated the RSF model's principle and Chan's global optimization idea and could get the global optimal solution. Secondly, a fast numerical minimization scheme based on split Bregman iterative algorithm was employed for overcoming drawbacks of noise and others. Finally, the curve evolved to the target boundaries quickly and accurately. The approach was applied in real special sea ice SAR images and synthetic images with noise, fuzzy boundaries and intensity inhomogeneity, and the experiment results showed that the proposed approach had a better performance than the edge detection methods based on the GMAC model and RSF model. The validity and robustness of the proposed approach were also verified in the experiment.

The rest of this study was organized as follows. Section 2 defined the proposed global optimization convex energy model based on RSF model and GMAC model. Section 3 introduced a fast minimization scheme based on split Bregman iterative algorithm for the proposed edge detection energy model and described in detail the iterative process of the numerical minimization. Section 4 presented the experiment results and evaluated the validity of our approach using real sea ice SAR images and synthetic images. Finally, Section 5 was a summary.

\section{GLOBAL OPTIMIZATION CONVEX ENERGY MODEL}

Currently, the edge detection based on the partial differential equation method has become a research focus. The first step of the partial differential method establishes an energy function model making use of the image information and some special requirements, and the key step is the solution of minimizing the energy function by means of variational method. But many existing variational image processing models are facing a common problem, i.e. being prone to get the local minima in minimization (which is not a global minimum) [3]. Variational energy function models Such as MS model [13], ACWE model [9] and RSF model [12] are non-convex. According to the optimization theory, non-convex energy function models may have local minima in minimization, which can stop the curve moving to the target boundaries and obtain unsatisfied edge detection results.

In this section, we defined a generalized convex function variational model which could get the global optimal solution, incorporating the RSF model's advantage [12] and Chan's global optimization idea [3]. The global optimization convex energy model was defined as follows.

\subsection{The RSF Model}

In order to solve the drawbacks caused by noise and intensity inhomogeneity, Li et al. [12] proposed RSF model. The RSF model used the local intensity information at a variable scale and got a great success in dealing with intensity inhomogeneity. Two fitting functions $f_{1}(x)$ and $f_{2}(x)$ locally approximated the intensities outside and inside the contour, and then were introduced in the RSF model. The energy function of the RSF Model was defined as follows:

$$
\begin{gathered}
\varepsilon^{R S F}\left(\phi, f_{1}(x), f_{2}(x)\right)=\lambda_{1} \int\left[\int_{\text {ousside }(c)} K_{\sigma}(x-y)\left|I(y)-f_{1}(x)\right|^{2} H(\phi(y)) d y\right] d x \\
+\lambda_{2} \int\left[\int_{\text {inside }(c)} K_{\sigma}(x-y)\left|I(y)-f_{2}(x)\right|^{2}(1-H(\phi(y))) d y\right] d x \\
+v \int|\nabla H(\phi(x))| d x+\mu \int \frac{1}{2}(|\nabla \phi(x)|-1)^{2} d x
\end{gathered}
$$

where $\lambda_{1}, \lambda_{2}, v$ and $\mu$ are weighting positive constants and $K_{\sigma}$ is a Gaussian kernel function with standard deviation $\sigma$. Two fitting functions $f_{1}(x)$ and $f_{2}(x)$ were defined as follows:

$$
\begin{gathered}
f_{1}(x)=\frac{K_{\sigma}(x) *[\phi(x) I(x)]}{K_{\sigma}(x) * \phi(x)} \\
f_{2}(x)=\frac{K_{\sigma}(x) *[(c-\phi(x)) I(x)]}{K_{\sigma}(x) *(c-\phi(x))}
\end{gathered}
$$

where the size of constant matrix $\mathrm{c}$ is the same as the given image, and the value of $\mathrm{c}$ is 1 .

\subsection{Chan's GMAC model}

Many existing variational image processing models are non-convex; therefore they are facing a common and serious drawback that they are prone to get the local minima in minimization (which is not a global minimum). According to the optimization theory, the local minima of edge detection models often stop the curve moving to the target boundaries and obtain completely wrong levels of detail and unsatisfied edge detection results in the end. Many solution schemes of variational models often use the gradient descent method, but that method often makes the results of edge detection depend on the initial position of the contour [3].

In the study of Chan [3], a global minimization active contour (GMAC) model for the piecewise constant segmentation was proposed. The model was described as follows.

$$
\text { For two given fixed } c_{1}, c_{2} \in \mathfrak{R} \text {, a global }
$$
minimizer of MS model $\left(\cdot, c_{1}, c_{2}\right)$ could be found using the following convex minimization formula:

$$
\begin{gathered}
\min _{0 \leq u \leq 1} \int_{D}|\nabla u|+\lambda \int_{D}\left\{\left(c_{1}-f(x)\right)^{2}-\left(c_{2}-f(x)\right)^{2}\right\} u(x) d x \\
\text { where } \sum=\{x: u(x) \geq \mu\} \text { for a.e. } \mu \in[0,1] .
\end{gathered}
$$




\subsection{Global Optimization Convex Energy Model} based on the RSF and GMAC

In this subsection, a global optimization convex energy model which introduced the region information fitting term of RSF model into a global minimization active contour model (GMAC) was defined [9]. The new model could get a global minimum and deal with some special images which were contaminated with noise and intensity inhomogeneity. The global optimization convex energy function was defined as follows:

$$
\begin{aligned}
\min _{0 \leq u \leq 1}\left\{E\left(u, f_{1}, f_{2}\right)=\right. & \int_{\Omega} g(x, y)|\nabla u| d x d y+\int_{\Omega} \lambda\left\{\int_{\Omega_{2}} K_{\sigma}\left|I(x, y)-f_{2}(x, y)\right|^{2} u d x d y\right. \\
& \left.\left.-\int_{\Omega_{1}} K_{\rho}\left|I(x, y)-f_{1}(x, y)\right|^{2} u d x d y\right\}\right\}
\end{aligned}
$$

where the first term in equation (4) is called weighted total variational (TV) term. To keep the geometry of the original features better and get a good edge detecting result, an edge indicator function $g(x, y)$ was introduced into the TV term, denoted

$$
g(x, y)=1 / 1+|\nabla I(x, y)|^{2} \quad(0<g(x, y) \leq 1)
$$
second term and the third term in equation (4) were called region fitting energy terms which drew on RSF model's advantage. $K_{\sigma}$ is called region scalability factor which is a Gaussian kernel function with standard deviation $\sigma ; K_{\sigma}$ is crucial to deal with intensity inhomogeneity; $\lambda$ stands for positive constants. Two fitting functions $f_{1}$ and $f_{2}$ defined in equation (4) were similar to those in equation (2).

For given fixed values of fitting functions $f_{1}(x), f_{2}(x) \in R$ (outside and inside the contour), a global minimizer was obtained according to the theorem proposed by Chan et al. [3].

\section{SPLIT BREGMAN ALGORITHM FOR MINIMIZATION OF GLOBAL OPTIMIZATION CONVEX ENERGY MODEL}

In numerical minimization, the method based on the gradient descent and Euler-Lagrange is often used $[3,12]$, but many experiments have shown that this numerical minimization method will cause the regularized process of the total variational to slow down, and this numerical minimization has also higher computational complexity and time complexity $[7,9,17]$. So Bresson proposed a numerical minimization method using a dual formulation which improves the convergence speed of the curve while reducing the complexity of the algorithm [1]. Then Goldstein and Osher proposed a fast minimization scheme based on split Bregman iteration technique for image segmentation. Experimental results have shown that computational validity of minimization scheme based on split Bregman is better than the methods of the dual formulation and graph optimization [14, $18,19]$.
Because the proposed optimization convex energy function included the L1 norm, it is ensured that the split Bregman algorithm could be used to solve the numerical minimization of energy function (4) quickly and accurately. The solving process of minimization was described in the following steps.

Firstly, the global optimization convex energy function for the edge detection was simplified by the following formula:

$$
\min _{0 \leq \phi \leq 1}|\nabla u|_{g}+<u, r>
$$

where

$$
\left\{\begin{array}{l}
|\nabla u|_{g}=\int_{\Omega} g(x, y)|\nabla u| d x d y \\
r=\lambda \sum_{i=1}^{2}(-1)^{i}\left|I_{i}(x, y)-f_{i}\right|^{2} \\
<u, r>=\int_{\Omega} r u(x, y) d x d y
\end{array}\right.
$$

Then an auxiliary variable $d \leftarrow \nabla u$ was introduced into constrained minimization problem (5). After that, a quadratic penalty function was added into equation (5) to convert the constrained minimization problem into an unconstrained problem, and the following optimization model was obtained.

$$
\left\{\begin{array}{l}
\arg \min _{0 \leq u \leq 1, d}|d|_{g}+<u, r>+\frac{\mu}{2}\left\|d-\nabla u-b^{k}\right\|^{2}, k>0 \\
b^{k+1}=b^{k}+\nabla u^{k+1}-d^{k+1}
\end{array}\right.
$$

According to the theory of variable separable, the optimal solution of equation (7) should satisfy the following expression:

$$
\Delta u=\frac{1}{\mu} r+\operatorname{div}\left(d^{k}-b^{k}\right), \quad 0<u<1
$$

Then Gauss Seidel iterative method was used to get an approximate solution of $u$ :

$$
\left\{\begin{array}{l}
\alpha_{i, j}=d_{i-1, j}^{x, k}-d_{i, j}^{x, k}-b_{i-1, j}^{x, k}+b_{i, j}^{x, k}+d_{i, j-1}^{y, k}-d_{i, j}^{y, k}-b_{i, j-1}^{y, k}+b_{i, j}^{y, k} \\
\beta_{i, j=} \frac{1}{4}\left(u_{i-1, j}^{k}+u_{i+1, j}^{k}+u_{i, j-1}^{k}+u_{i, j+1}^{k}\right)-\frac{r}{\mu}+\alpha_{i, j} \\
u_{i, j}^{k+1}=\max \left\{\min \left\{\beta_{i, j}, 1\right\}, 0\right\}
\end{array}\right.
$$

According to the variational method, the following formula was used to minimize the optimization problem (7) with respect to $\stackrel{\text { ut }}{d}$ :

$$
d^{k+1}=\frac{\nabla u^{k+1}+b^{k}}{\left|\nabla u^{k+1}+b^{k}\right|} \max \left(\left|\nabla u^{k+1}+b^{k}\right|-\frac{1}{\mu}, 0\right)
$$

Based on the description of the above simple solution, the process of the minimization based on split Bregman iterative algorithm was described as follows:

Begin

Step1. Initialization:

$u_{0}=\frac{I(x, y)}{\max (I(x, y))}$

Compute initial $f_{1}$ and $f_{2}$ by Eq. (2) 
Repeat

Step2. Iteration:

While $\left\|u^{k+1}-u^{k}\right\|>\varepsilon$ do

Define $r^{k}$ by Eq. (9)

Update $u^{k+1}$ by Eq. (12)

$d^{k+1}=\frac{\nabla u^{k+1}+b^{k}}{\left|\nabla u^{k+1}+b^{k}\right|} \max \left(\left|\nabla u^{k+1}+b^{k}\right|-\frac{1}{\mu}, 0\right)$

$b^{k+1}=b^{k}+\nabla u^{k+1}-d^{k+1}$

Update $f_{1}$ and $f_{2}$ by Eq. (2)

End

\section{EXPERIMENT RESULTS AND DISCUSSION}

The proposed approach was applied in the real sea ice SAR images and synthetic images in this section. These experiment images were contaminated with noise, fuzzy boundaries and intensity inhomogeneity. We also presented the experimental results and evaluated the performance of the proposed approach compared with RSF model [12] and GMAC [3].

\subsection{Dataset}

To evaluate the performance of the proposed approach in this study, two groups of images were used for the experiment. The first group comprised 50 sea ice remote sensing SAR images and the size of the images was between $50 \times 120$ pixels and $258 \times 345$ pixels. The second group contained three synthetic images and the size of the images was between $75 \times 79$ pixels and $127 \times 96$ pixels.

\subsection{Parameter Setting}

The parameters in the proposed approach were chosen empirically. Specifically, $\lambda$ was set as 0.0001 , and $\mu=1.0$. In order to balance against edge detection precision and acceptable convergence speed, iteration termination threshold $\varepsilon=1 e-2$ was chosen. The value of scale parameter $\sigma$ was chosen later by hand.

\subsection{Experimental results and Comparisons}

In the first experiment, the edge detection results were shown for four typical sea ice SAR images using the proposed algorithm in Fig.1. The first row shows the original images, and the second row shows the edge detection results applying the proposed approach. It can be seen clearly from the original sea ice SAR images that the contrast between sea ice and seawater is low, the contours of the sea ice were very obscure, and some regions in images are contaminated with intensity inhomgeneity, but we could still obtain the satisfactory results of edge detection using the proposed approach for these challenging sea ice SAR images. The first experiment showed that our approach was effective and robust to the sea ice SAR images contaminated with low contrast, high noise and intensity inhomogeneity. Moreover, the proposed algorithm had lower computational complexity and time complexity. The size of original image, the value of scale parameter, and the respond time of CPU for the images in Fig. 1 are given in Table 1. Therefore, we could further get the shape and acreage of sea ice, identify sea ice by an advanced classifier, and confirm the location of sea ice more easily and quickly using the obtained edge information of sea ice.
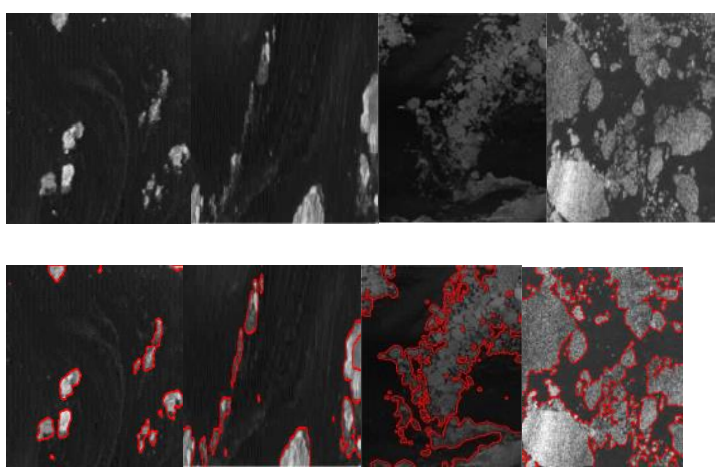

Fig.1. Experiments for four typical sea ice SAR images with noise, fuzzy boundaries and intensity inhomogeneity. Row 1: original synthetic images. Row 2: the results of edge detection obtained using the proposed approach.

Table1. The respond time of CPU using the proposed approach for four sea ice SAR images in fig. 1 with the

\begin{tabular}{|c|r|r|r|}
\hline Image & Size(pixel) & $\sigma$ & CPU time(s) \\
\hline Image1 & $261 \times 222$ & $\sigma=3$ & 13.5 \\
Image2 & $432 \times 161$ & $\sigma=2$ & 32.8 \\
Image3 & $258 \times 345$ & $\sigma=5$ & 28.2 \\
Image4 & $189 \times 206$ & $\sigma=6$ & 19.1 \\
\hline
\end{tabular}

In the second experiment, the edge detection results for three synthetic images are shown in Fig. 2 . In order to demonstrate the accuracy and validity of the proposed approach, we gave the comparisons of the proposed approach with two important approaches: RSF model [12] and GMAC model [3]. Row1 shows the original synthetic images. The edge detection results using the proposed approach, RSF model and GMAC model, are shown in row 2, row 3 and row 4 respectively. It can be clearly seen that the first synthetic image in Fig. 2 is contaminated with strong noise and has various shapes including an interior contour, the second synthetic image in Fig. 2 is contaminated with severe intensity inhomogeneity, and the last synthetic image in Fig. 2 has low contrast, weak boundaries and severe intensity inhomogeneity. For the first synthetic image with strong noise, the edge detection results using three approaches were satisfactory. Nevertheless, it was found that the proposed approach could get more precise the 
results of edge detection and had a better ability to suppress strong noise. For the second and third synthetic images contaminated with weak boundaries and severe intensity inhomogeneity, both our approach and RSF model could obtain ideal edge detections. However, the GMAC model could only get the failure edge detection results no matter what parameters were selected. It was because that GMAC model only used the global intensity information of the image so that cannot deal with the images contaminated with intensity inhomgeneity.
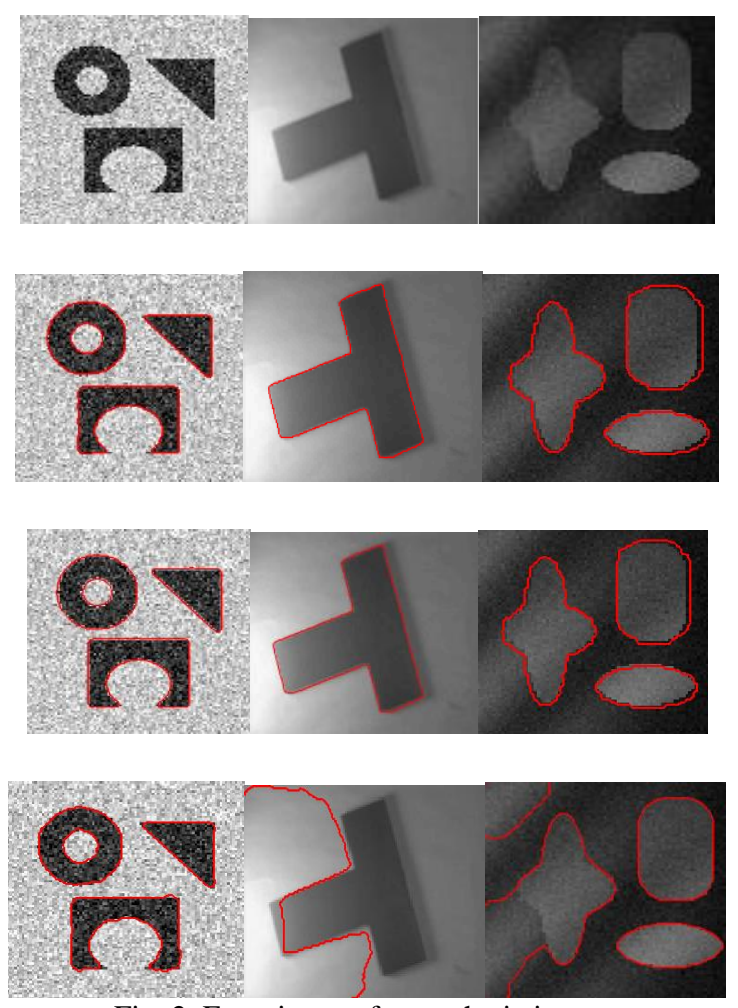

Fig. 2. Experiments for synthetic images contaminated with intensity inhomogeneity, strong noise, low contrast and fuzzy boundaries. Row 1: original synthetic images. Row 2: the results of edge detection using the proposed approach. Row 3: the results of edge detection using RSF model. Row 4: the results of edge detection using GMAC model

\subsection{Computational performance of the proposed approach}

The accuracy of edge detection in Fig. 2 was similar between the proposed approach and RSF model. However, RSF model is non-convex, so it is prone to get the local minima in minimizing the energy function to stop the curve from moving to real boundary of the target [9]. Moreover, the computational procedures of the proposed approach were more efficient compared to RSF model. It was because that a fast minimization scheme based on split Bregman iterative algorithm was used in the proposed approach in the numerical minimization, while the energy functions of the other two models used the conventional gradient descent method. The size of original image, the value of scale parameter $\sigma$ and the respond time of CPU for the images in Fig. 2 are shown in Table 2. During the experiments, the response time of $\mathrm{CPU}$ was recorded using Matlab codes in Matlab R2007b running on PC (Intel i3-2310M, CPU 2.10GHz, 4GB RAM, Windows 7). If a better programming language was used for recording the proposed approach efficiently, the run time could be significantly reduced.

Table 2. Comparison of the respond time of CPU between the proposed approach and other models for three synthetic images in fig. 2 with the same order

\begin{tabular}{|c|r|r|r|r|r|r|}
\hline & & \multicolumn{4}{|c|}{ CPU time(s) } \\
\cline { 3 - 7 } Image & Size(pixel) & $\begin{array}{r}\text { The } \\
\text { proposed } \\
\text { algorithm }\end{array}$ & $\begin{array}{r}\text { RSF } \\
\text { Model }\end{array}$ & $\begin{array}{r}\text { GMAC } \\
\text { model }\end{array}$ \\
\hline Image1 & $100 \times 100$ & $\sigma=4$ & 1.3 & $\sigma=4$ & 2.8 & 1.9 \\
Image2 & $75 \times 79$ & $\sigma=3$ & 1.8 & $\sigma=3$ & 3.5 & 2.4 \\
Image3 & $127 \times 96$ & $\sigma=3$ & 2.6 & $\sigma=3$ & 8.3 & 5.1 \\
\hline
\end{tabular}

\section{CONCLUSION}

In this study, a general and fast edge detection approach based on global optimization convex model and Split Bregman minimization algorithm was put forward. The proposed approach could figure out the global optimal solution of the energy function by incorporating the RSF concept and the Split Bregman minimization algorithm into GMAC model. In the numerical minimization, we used a fast iterative algorithm based on split Bregman algorithm that made use of a splitting technology to split the L1 term, thereby making the numerical minimization much simpler and more quickly. The proposed approach was applied in real sea ice SAR images and synthetic images contaminated with intensity inhomgeneity and noise, and the experiment results showed that the proposed approach had a better performance than the edge detection methods based on the GMAC model and RSF model. The validity and robustness of the proposed approach were also verified in the experiment. In the future, we will further improve the adaptive selection of the scale parameter $\sigma$.

\section{SOURCE OF FUNDING}

This work is supported by the National Natural Science Foundation of China (No. 61501082 and No.61502435), Humanities and Social Sciences Project of MOE (Ministry of Education in China) (No.15YJCZH005), Natural Science Foundation Project of Liaoning Province (No.L2015020017, No.L20170540232 and No.L20170540230), Scientific Research Project of Liaoning Province Educational Department (No.L2015137) and Social Science Planning Foundation Project of Liaoning Province (No. L15CGL009). 


\section{REFERENCES}

1. Bresson X, Chan T. Fast dual minimization of the vectorial total variation norm and applications to color image processing. Inverse Problems and Imaging 2008; 2(4): 455-484.

http://dx.doi.org/10.3934/ipi.2008.2.455

2. Chan T, Vese L. Active contours without edges. IEEE Transactions on Image Processing 2001; 10(2): 266-277. http://dx.doi.org/10.1109/83.902291

3. Chan T. Algorithms for finding global minimizes of image segmentation and denoising models. SIAM J. Applied Mathematics 2006; 66(5): 1632-1648. http://dx.doi.org/10.1137/040615286

4. Clausi DA, Yue B. Comparing co-occurrence probabilities and Markov random fields for texture analysis of SAR sea ice imagery. IEEE Transaction on Geoscience and Remote Sensing 2004; 42(1) http://dx.doi.org/10.1109/TGRS.2003.817218

5. Cremers D, Rousson M, Deriche R. A review of statistical ap-proaches to level set segmentation: Integrating color, texture, motion and shape. Int. J. Comput. 2007; 72(2): 195-215. https://dx.doi.org/10.1007/s11263-006-8711-1

6. Goldstein $\mathrm{T}$, Bresson $\mathrm{X}$, Osher S. geometric applications of the split bregman method: segmentation and surface reconstruction. Journal of Scientific Computing 2010; 45(1-3): 272-293. http://dx.doi.org/10.1007/s10915-009-9331-z

7. Gui J, Rao X, Ying Y. Fruit shape detection by level set. Journal of Zhejiang University Science A 2007; 8(8):1232-1236 http://dx.doi.org/10.1631/jzus.2007.A1232

8. He L, Peng Z, Everding B, Wang X, Han C, Weiss K, Wee W. A comparative study of deformable contour methods on medical image segmentation. Image Vis. Comput. 2008; 26(5): 141-163. https://doi.org/10.1016/j.imavis.2007.07.010

9. Jing Y, An JB, Liu ZX. A novel edge detection algorithm based on global minimization active contour model for oil slick infrared aerial image. IEEE Transaction on Geoscience and Remote Sensing, 2011; 49(6): 2005-2013. http://dx.doi.org/10.1109/TGRS.2010.2103671

10. Jing Y, An JB, Wang YX, Liu Z X, Liu JX. A New Region-Based Active Contour Edge Detection Algorithm for Oil Spills Remote Sensing Image. Journal of Convergence Information Technology 2012; 7(21): 112-119.

11. Kwon TJ, Li J, Wong A. ETVOS: An Enhanced Total Variation Optimization Segmentation Approach for SAR Sea-Ice Image Segmentation. IEEE Transactions on geoscience and remote sensing 2013; 51(2):925-934.

http://dx.doi.org/10.1109/TGRS.2012.2205259

12. Li C M, Kao C, Gore J. Minimization of regionscalable fitting energy for image segmentation. IEEE Transactions on Image Processing 2008; 17(10): 1940-1949.

http://dx.doi.org/10.1109/TIP.2008.2002304

13. Mumford D, Shah J. Optimal approximations by piecewise smooth functions and associated variational problems. Communications on Pure and Applied Mathematics 1989; 42(5): 577-685. http://dx.doi.org/10.1002/cpa.3160420503

14. Osher S, Burger M, Goldfarb D, Xu J, Yin W. An iterative regularization method for total variation- based image restoration. Multiscale Model. Simul. 2005; 4: 460-489.

http://dx.doi.org/10.1137/040605412

15. Torre V, Poggio T. On Edge Detection. IEEE Transactions on Pattern Analysis and Machine Intelligence, 1986; 8(2): 147-16. http://dx.doi.org/10.1109/TPAMI.1986.4767769

16. Wang L, Li C, Sun Q, Xia D, Kao C. Active contours driven by local and global intensity fitting energy with application to brain MR image segmentation. J. Comput. Med. Imaging Graph. 2009; 33 (7): 520531.

https://doi.org/10.1016/j.compmedimag.2009.04.010

17. Wu QG, An JB, Lin B. A Texture Segmentation Algorithm Based on PCA and Global Minimization Active Contour Model for Aerial Insulator Images. IEEE Journal of Selected Topics in Applied Earth Observations and Remote Sensing 2012; 5(5): 15091518.

http://dx.doi.org/10.1109/JSTARS.2012.2197672

18. Yang YY, Zhao Y, Wu BY. Split Bregman Method for Minimization of Fast Multiphase Image Segmentation Model for Inhomogeneous Images. J Optim Theory Appl 2015; 166: 285-305. http://dx.doi.org/10.1007/s10957-014-0597-4

19. Yin W, Osher S, Goldfarb D, Darbon J. Bregman iterative algorithms for L1-minimization with applications to compressed sensing. SIAM J. Imaging Sci. 2008; 1(1): 143-168. http://dx.doi.org/10.1137/070703983

Received 2017-12-13

Accepted 2018-02-19

Available online 2018-03-01

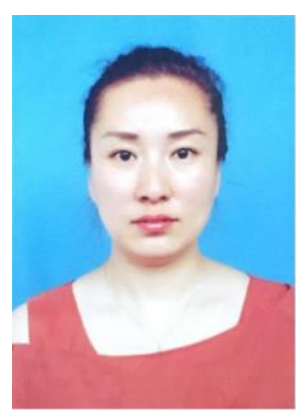

Yu JING was born in Liaoyang city of Liaoning province in 1982. In 2005, she was working toward the Ph.D. degree at information science and technology institute of Dalian Maritime University, in Dalian city of Liaoning province. She received the Ph.D. degree in computer application technology in 2011. She currently works as an associate professor in Software College of Dalian Foreign Language University. Her research interests include remote sensing image processing, pattern recognition, and computer vision. Now, she is undertaking wo research projects supported by the Scientific Research Project of Liaoning Province Educational Department and National Natural Science Foundation of China (NSFC). Furthermore, up to now, she has participated in over 10 provincial level research projects as a principal member.

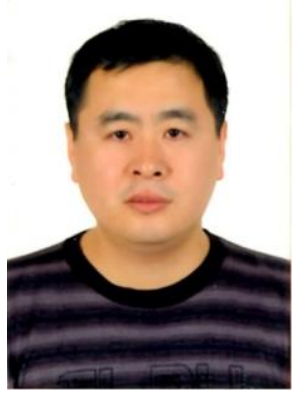

Jianxin LIU was born in Zhuanghe city of Liaoning province in 1981. In 2005, he was working toward the master's degree at information science and technology institute of Dalian Maritime University, in Dalian city of Liaoning province. He received the master's degree in computer application technology in 2008 . He is now a 
lecturer in Software College of Dalian Foreign Language University. His research interests include artificial intelligence and image processing. Up to now, he has participated in multiple provincial level research projects as a principal member.

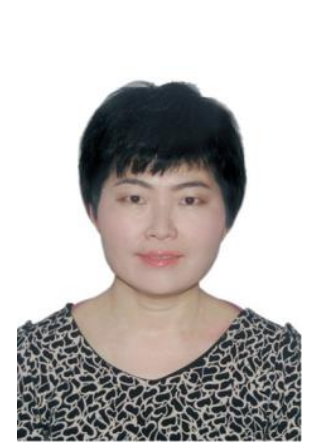

Zhaoxia LIU was born in Jinan city of Shandong province in 1982. In 2005, she was working toward the Ph.D. degree at information science and technology institute of Dalian Maritime University, in Dalian city of Liaoning province. She received the $\mathrm{Ph} . \mathrm{D}$. degree in computer application technology in 2011. She is of Dalian Foreign Language University. Her research interests include remote sensing image processing, image registration technique, pattern recognition, and computer vision. She has undertaken two research projects supported by the Natural Science Foundation Project of Liaoning Province and National Natural Science Foundation of China (NSFC). Furthermore, she has participated in a lot of provincial level research projects as a principal member.

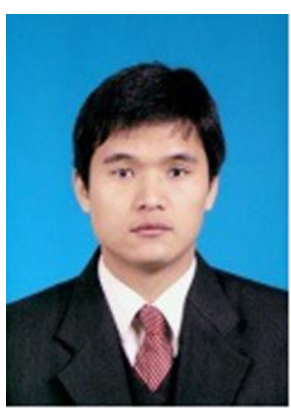

Hongju CAO was born in Yuncheng County of Shandong Province in 1980. $\mathrm{He}$ is currently a lecturer in the Software College of Dalian University of Foreign Languages. He received the M.S. degree in Operation Research and Control Theory form the Dalian University of Technology in 2006. $\mathrm{He}$ is currently pursuing the $\mathrm{Ph} . \mathrm{D}$. degree in Information Science and Technology Institute of Dalian Maritime University. His research interests include theory and algorithms of optimization and hyperspectral images processing. 